\title{
Perceptual-motor deficits in children with Down syndrome: Implications for intervention
}

\author{
Naznin Virji-Babul', Kimberly Kerns², Eric Zhou', Asha Kapur ${ }^{3}$ and Maggie \\ Shiffrar ${ }^{4}$ \\ 'Down Syndrome Research Foundation, Burnaby, BC. University of Victoria, Victoria, B.C. \\ ¿University of Victoria, Victoria, B.C. \\ ${ }^{3}$ Wake Forest University, North Carolina \\ ${ }^{4}$ Rutgers University
}

\begin{abstract}
Early intervention approaches for facilitating motor development in infants and children with Down syndrome have traditionally emphasised the acquisition of motor milestones. As increasing evidence suggests that motor milestones have limited predictive power for long-term motor outcomes, researchers have shifted their focus to understanding the underlying perceptual-motor competencies that influence motor behaviour in Down syndrome. This paper outlines a series of studies designed to evaluate the nature and extent of perceptual-motor impairments present in children with Down syndrome. 12 children with Down syndrome between the ages of 8-15 years with adaptive ages between 3-7 years (mean age $=5.6$ years $+/-1.45$ years) and a group of 12 typically developing children between the ages of $4-8$ years (mean age $=5.4+/-1.31$ years) were tested on their ability to make increasingly complex perceptual discriminations of motor behaviours. The results indicate that children with Down syndrome are able to make basic perceptual discriminations but show impairments in the perception of complex visual motion cues. The implications of these results for early intervention are discussed.
\end{abstract}

Keywords: Perceptual-motor behaviour, biological motion, object motion, emotive human movement

\section{Introduction}

It is generally accepted that motor impairments are inevitably present, to greater or lesser extents, in children with Down syndrome. While the complex relationships between the genotype and phenotype of Down syndrome have yet to be elucidated, evidence suggests that developmental outcomes are influenced by the interactions between individuals' genetic tendencies and their environment (Flint, 1999; Nokelainen \& Flint, 2004). In spite of this, it remains unclear whether 'enriched environments', in the form of motor-based intervention programs, impact on longterm developmental motor outcomes in Down syndrome (Mahoney et al., 2004; Spiker \& Hopmann, 1997).

The limited success of motor based interventions may be due, in part, to the influence of traditional approaches in developmental psychology that emphasise achievements and comparisons of developmental milestones with typically developing children. A large body of literature has documented delays in basic motor skills, such as walking, reaching and grasping, in children with Down syndrome (Carr,
1970; Haley, 1986; Palisano et al., 2001). Much of this developmental delay continues to be attributed to isolated factors such as low muscle tone (Harris, 1985). Yet, recent research indicates that muscle tone evaluated under passive conditions may have little relationship with the strategies used by the nervous system under more dynamic conditions such as during standing (Webber, Virji-Babul et al. 2004). In fact, Latash (2000) has suggested that over time, individuals with Down syndrome learn to develop adaptive motor strategies that optimise safety and stability. He suggests that early intervention focus on exploration of a range of motor solutions to avoid the development of fixed patterns of motor behaviour. Nevertheless, the focus of many early intervention programs continues to be on facilitating motor skills within the confines of typical developmental trajectories without considering the broader context of functional motor behaviour.

It is important to recognise that the motor system does not function in isolation (e.g., Cole, 1995; Grezes \& Decety, 2001). Instead, perceptual processes interact with motor 
processes during action production, action correction, and action comprehension (e.g., Shiffrar \& Pinto, 2002; Wilson \& Knoblich, 2005). Importantly, it is not yet known how the pathophysiology of Down syndrome is related to the development of perceptual-motor behaviour. Nadel (1999) reported that differences in brain structure begin to emerge in the first few months of life in infants with Down syndrome. These differences include reduced volume in the frontal cortex, superior temporal gyrus, brainstem, cerebellum and hippocampus. Some of these areas are involved in the visual analysis of human motion (Stevens et al., 2000; Grossman \& Blake, 2002). While the motor consequences of volumetric differences in these neural differences have not been well established, it is reasonable to hypothesise that these differences may impact on the mechanisms involved in processing perceptual motor information.

\section{Perceptual-motor coupling in children with Down syndrome}

There is already some evidence in the literature that children with Down syndrome demonstrate impairments in perceptual-motor coupling. For example, when children with Down syndrome perform motor tasks requiring anticipatory actions (such as catching), their impairments appear to be attributable to difficulties in regulating the temporal aspects of their actions (Henderson et al., 1981; Savelsbergh et al., 2000). Charlton et al. (2000) reported that children with Down syndrome have difficulty in properly adjusting both the spatial and temporal aspects of their grasp as a function of object size or task goal. They suggest that difficulties in the use of the perceived object properties in action planning may point to a dysfunction in relating information about limb position with respect to the environment to task demands.

One experimental approach that has been used to study the relationships between visual perception and motor output involves the modification of anticipatory gaits during obstacle crossing. Walking in the natural environment often involves anticipatory adaptations to either avoid or negotiate obstacles. Recently, Virji-Babul and Brown (2004) examined the movement strategies used by young children with Down syndrome as they crossed obstacles of two different heights - a 'subtle' obstacle that was placed at a very minimum height off the floor ( $1 \%$ of total body height) and an 'obvious' obstacle that was placed at a much higher height off the floor (15\% of total body height). Spatial and temporal measures of the gait cycle were analysed. Children with Down syndrome showed a robust scaling of toe elevation to obstacle height, implying that they were able to successfully extract information about obstacle height and appropriately match this information to their movements. However, visual information about the obstacle was not used consistently to modulate movements early in the gait cycle. Greater step length variability was observed in response to the subtle obstacle suggesting that some form of anticipatory adjustments were being made. In contrast, there was very little variability observed in response to the higher obstacle. This finding, in combination with the observation that children with Down syndrome stopped in front of the higher obstacle for long periods of time, indicated that on-line anticipatory adjustments could not be made effectively and as a result, the children maintained their 'typical' gait pattern. This suggests that children with Down syndrome maybe unable to use early visual cues about an obstacle and so wait until they reach an obstacle to extract the visual information needed to appropriately modulate their actions. This conclusion corroborates the findings of Charlton et al. (2000) and others and provides further evidence of difficulties in perceptual-motor coupling in Down syndrome.

The studies described below used a different technique to investigate perceptual-motor coupling in Down syndrome. This technique, based on the pioneering studies of Johansson (1973), provides a means by which the static form (contextual) cues defining objects and bodies are minimised while motion cues are retained. In his classic studies, Johansson placed luminous patches on the major joints and head of human models and filmed these models so that only these 'point-lights' were visible as small dots (see Figure lA for an example). When static, these displays appear as relatively meaningless configurations of illuminated points against a dark screen. However, when set in motion, these points rapidly transform into a coherent display of a moving actor. Research has since shown that adults can accurately identify actions (Johansson, 1975), gender (Pollick et al., 2002), identity (Loula et al., 2005) and emotional states (Dittrich et al., 1996) from the bodily movements defined in these dynamic point-light displays. Indeed, children as young as 3 years of age are able to recognise and differentiate human from non-human motions depicted in point-light displays (Pavlova et al., 2001). Furthermore, by three months of age, children begin to discriminate different types of point-light actions (Booth et al., 2002; Fox \& McDaniel, 1982). Since neither adults nor children are able to accurately interpret static depictions of point-light displays (Johansson, 1973; Pavlova et al., 2001) motion must be a critical parameter for recognition of both human and non-human forms.

Research using this approach has been instrumental in revealing the interactions between the perceptual and motor systems across developmental stages and, to some extent, in individuals who have specific visual/perceptual-motor impairments. For example, Moore et al. (1997) tested individuals with autism, age-matched control participants, and non-autistic retarded individuals on their visual analysis of point-light displays. They reported that all three groups were equally accurate in their ability to distinguish between biological and non-biological motions (i.e., bouncing ball). Other evidence suggests that autistic children may nonetheless demonstrate relative impairments in the detection of point-light displays of human action (Blake et al., 2003). Furthermore, individuals with autism appear to be significantly impaired in their ability to discriminate between point-light depictions of different emotional states while non-autistic, mentally retarded children are not impaired (Moore et al., 1997). 
To our knowledge, this approach has not been used to analyse the perceptual-motor coupling of individuals with Down syndrome. We therefore set out to examine the visual analysis of point-light displays of human and object motions in a group of young children with Down syndrome. The goals of our study were three fold. Our first goal was to determine if children with Down syndrome can perform basic perceptual discriminations between human motion and object motion. The second goal was to evaluate the ability of children with Down syndrome to process more complex emotional stimuli. Finally, the third goal was to determine if children with and without Down syndrome differ in their abilities to perceptually discriminate between complex motoric stimuli.

\section{Experiment I}

\section{Human motion vs object motion}

The purpose of this experiment was to determine if children with and without Down syndrome can accurately discriminate between point light displays of human and object motion. Previous research has suggested that the visual analysis of human motion and object motion systematically differ (Shiffrar \& Pinto, 2002). The differences are thought to reflect differential activation of and contributions by the motor system during the visual analysis of human motion (e.g., Stevens et al., 2000). As outlined in the introduction, children with and without Down syndrome exhibit systematic differences in their motor capabilities. Will these motor differences drive differences in their abilities to perceive human motion? To the extent that children are impaired in their perception of human motion, they should be compromised in their ability to discriminate between point-light displays of human and object motions.

\section{Method}

\section{Participants}

The Down syndrome group consisted of 12 children between the ages of 8-15 years. We assessed adaptive age by asking parents to complete the Scales of Independent Behavior - Revised (SIB-R; Bruininks, Woodcock, Weatherman \& Hill, 1996). This rating scale evaluates functional independence and adaptive function. The measure has four subtests - Motor, Social/Communication, Personal Living Skills and Community Living skills. An overall adaptive behaviour score is based on the average of the four subtests. Since IQ measures are based solely on cognitive skills, they have a minimal motor component and rely more heavily on verbal abilities (both receptive and expressive). While youngsters with Down syndrome often have verbal abilities similar to children of very young ages (2-3), their adaptive behaviour, or ability to act appropriately and problem solve within their daily life, is often similar to an older child. We therefore selected an 'age equivalence' based on adaptive function as a more reflective measure of children's motor skills. The scores on adaptive age for the Down syndrome group ranged between 3-7 years with a mean age of 5.6 years $(\mathrm{SD}=1.45)$. Therefore, a group of 12 typically developing children between the ages of 4-8 years was used as a comparison. The mean age of this group was 5.4 years $(S D=1.31$ years $)$. The same participants completed all three experiments.

\section{Stimulus construction}

A 3-D VICON motion capture system was used to capture or measure the motions of various actors and objects. To create the point-light actor or 'biological' displays, two typically developing children and two neurologically normal adults were asked to walk along a walkway at a comfortable speed. All the participants had reflective markers attached to the major joints of their bodies. These markers were then processed to create movies of 'point-light' displays against a dark background (Figure 1A). These displays of human walking depicted either 6 dots on the lower extremities (hip, knee and foot on both sides of the body) or 13 dots (head, shoulder, elbow, wrist and lower extremities). A total of 4 point-light movies consisting of four different point-light walkers (two with 6 points and two with 13 points) were created. To create the object or nonbiological stimuli, reflective markers were attached to 6 points of four objects - i.e. juggling balls, a wheel, a skipping rope, and a Frisbee (Figure 1B). The movements of these four objects
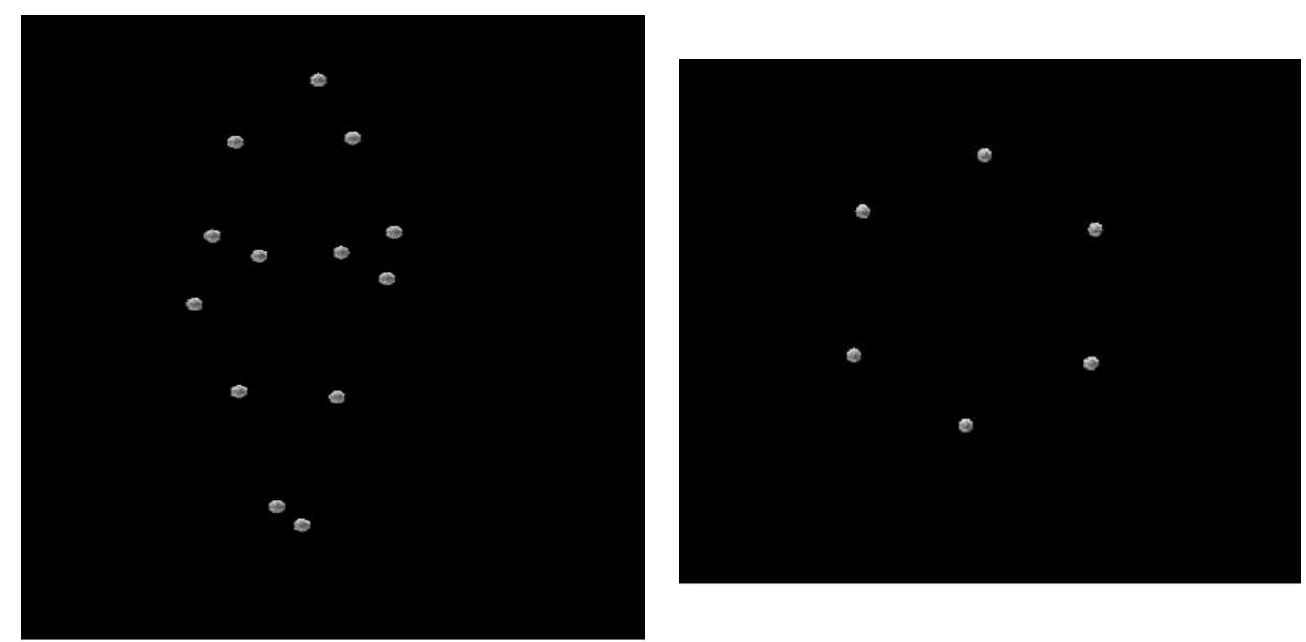

Figure I. (A) A single frame from a point-light display of human walking. While such displays are difficult to interpret when static, the coherent perception of human gait arises when they are set in motion. (B) A single frame from a point-light display of a rotating wheel. This represented one of four displays from the non-biological motion condition. 
were then captured and stimuli were constructed by the same means used with the biological stimuli. The duration of every movie was 6 seconds.

\section{Procedure}

A series of training displays was created to familiarise all participants with the point light displays. All participants were first trained with digital videos (full colour and form) that showed the actual motions of a child performing an action other than walking (i.e. twirling, kicking or jumping). The children were asked to observe the reflective markers on the joints of the individual and to respond to the question "What is that boy/girl doing?" Each participant was then shown a dynamic, point-light display paired with the corresponding digital (again, full colour and form) video recording of the same motion using a split screen (see Figure 2). The children were asked to observe the motion of the dots and compare these movements to those in the digital video display. Finally, each participant was shown only the point light display and asked to comment on what the subject was doing. The experiment began only after the participant clearly demonstrated that he/she understood the task and the relationship between the point light displays and the actual motions. In the experimental session, each participant viewed a sequential series of 8 separate point-light stimuli ( 4 human and 4 object motion) in a random order. Participants were instructed to report whether the dots in each movie showed a "person" or "not a person."

\section{Results}

An individual accuracy score was calculated for each participant. A point was given for each trial in which a correct response was given, yielding scores with a range from 0 to 8 with a mean of 4 expected by chance. The average accuracy score for the children with Down syndrome was 6 . An average of 7.5 was found for the typically developing children (Figure 3). An independent t-test was computed to determine if the responses of the two groups differed from one
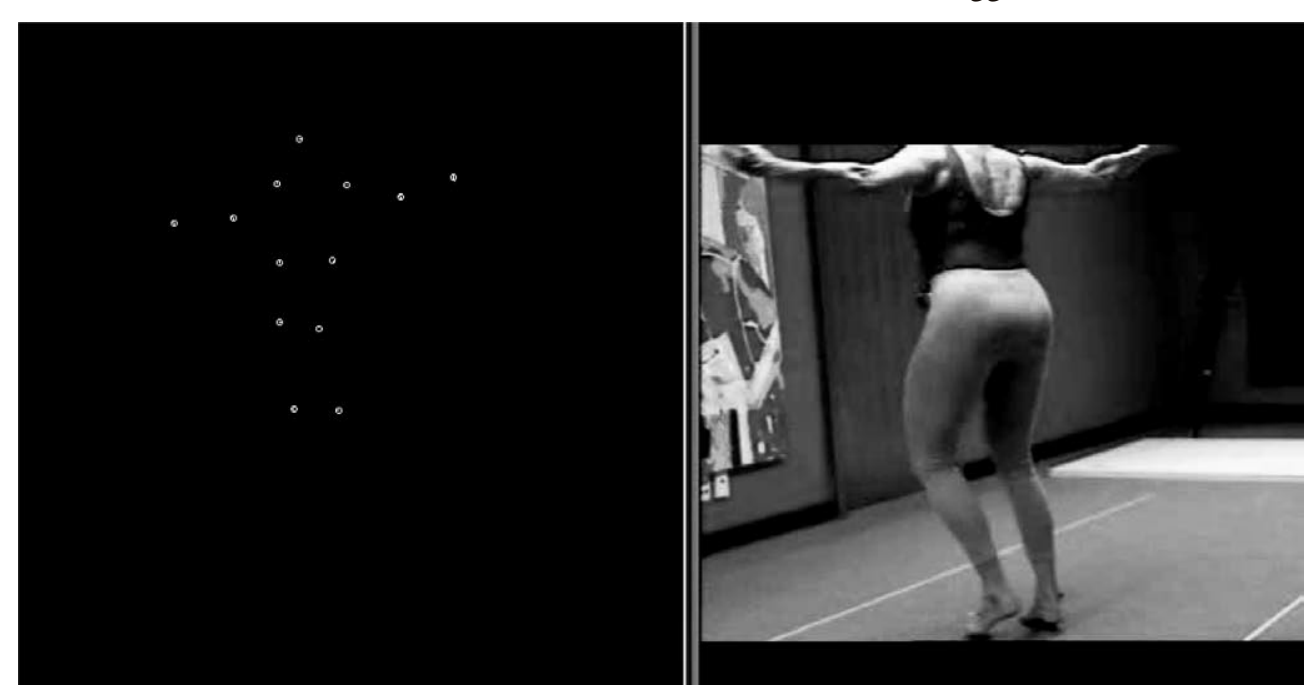

Figure 2. Split screen showing point-light display and corresponding full video display

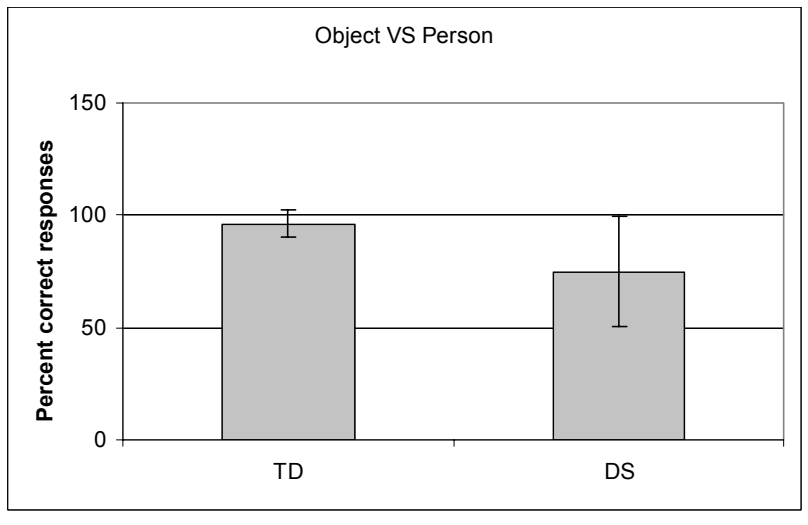

Figure 3. The results of Experiment I. The ability to perceptually differentiate point-light displays of human and object motions in typically developing children (TD) and children with Down syndrome (DS) was tested. Performance is described as the percentage of trials during which children accurately reported whether or not a person was present. Error bars depict the standard deviation.

another. The responses of the two groups were significantly different, $F(4,23)=3.327, p=.03$. The accuracy scores for each group were then compared with chance performance with a one-tailed, one-sample $t$ test. Both groups performed significantly above chance on this measure. Down syndrome group, $t(11)=3.7, p=.003$ typically developing group $t(11)=24, p<.0001$ indicating that they were able to discriminate human motion from non-human motion using point light displays and were not merely 'guessing' correctly.

\section{Discussion}

The results of this experiment indicate that children with Down syndrome can differentiate between the motions of people and the motions of inanimate objects. However, children with Down syndrome do not perform this discrimination as accurately as typically developing children. This suggests that children with Down syndrome are able to perceive and interpret point-light displays of human action.

\section{Experiment 2}

\section{Emotive human movement}

Having determined that children with Down syndrome are able to differentiate human and object motions at above chance levels, we were then able to examine the impact of Down syndrome on the perception of more complex attributes. Contrary to 
the popular perception of children with Down syndrome as easy, happy, and socially engaged, there is evidence that these children have specific impairments in social-cognitive development (Wishart \& Pitcairn, 2000; Kasari, Freeman \& Hughes, 2001). Previous research suggests that typically developed observers can determine an individual's emotional state from point-light displays of that person's actions (Dittrich et al., 1996; Heberlein et al., 2004). Recent data from our laboratory using the same displays also showed that typical adults are able to recognise emotional states from point-light displays with $93 \%$ accuracy (Kapur, Kapur, Virji-Babul et al., 2005). To determine whether children with Down syndrome can identify the emotional cues available in body movements, the following experiment was conducted.

\section{Method}

\section{Stimulus construction}

An experienced female dancer was recruited to perform expressive body movements representing the four target emotions of happiness, sadness, anger and fear. The dancer had 13 reflective markers attached to the major joints of her body. Sixteen movie clips, each approximately $20 \mathrm{sec}-$ onds in length, were created from the motion capture data. The resulting point-light stimuli were tested on a group of 10 typically developing preschool children. Of the original 16 point-light stimuli, the 8 stimuli that were consistently categorised by these children were selected for testing with Down syndrome children. An example of a point-light display showing a 'happy' action is shown in Figure 4.

\section{Procedure}

All the participants were first shown a sheet depicting four simple, drawn cartoon faces. Each face depicted one of the four possible emotions: happy, sad, mad, or scared. We first examined whether each participant could identify the four target emotions used in the displays. For the typically developing children, the experimenter pointed to each face and asked the child to describe the emotion depicted on the cartoon face. All of the typically developing children correctly labelled the target emotions. Children with Down syndrome were asked to point to the face that represented one of the four target emotions. All the children with Down syndrome were able to correctly indicate each emotion named.

Each child was then asked to view a point light movie clip. After viewing the point light display, the child was a given a forced-choice between two target emotions; for example, "is this person happy or sad"? An answer sheet with two cartoon faces per stimulus was placed in front of the child. Only one row of faces was visible during each trial. Children were asked to identify the cartoon face that best described the emotion depicted in the point-light movie. If the child was unable to respond verbally, he/she was asked to respond by pointing to the cartoon face.

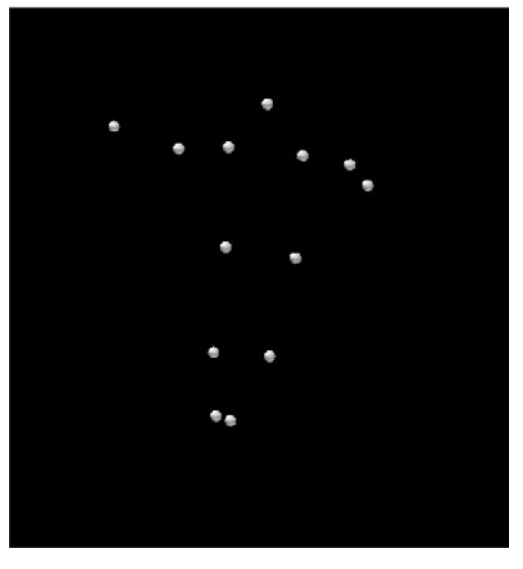

Figure 4. A single frame from a sample stimulus from Experiment 2. This frame illustrates a happy point-light actor.

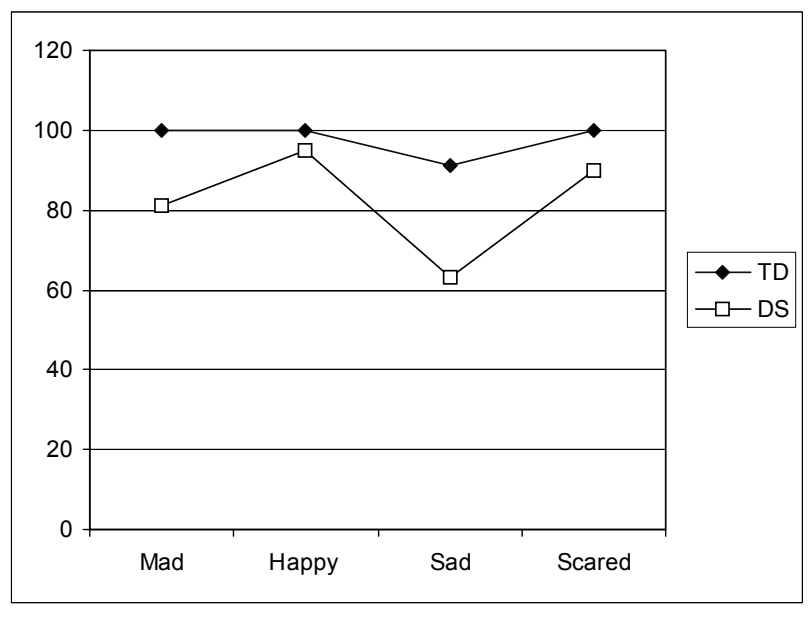

Figure 5. The results of Experiment 2. Typically developing children (TD) and children with Down syndrome (DS) identified the emotions depicted by point-light defined actors. Performance accuracy is broken down by the four emotions tested.

\section{Results}

An individual accuracy score was calculated for each participant in this emotion recognition task. A point was given for each trial in which the target emotion was correctly identified. The percentages of correct responses for each of the four emotions are illustrated in Figure 5. The average percent correct responses for the typically developing children were 100, 100, 91, 100 for the emotions of happy, sad, mad and scared respectively. For the children with Down syndrome, the average percent correct responses were 81 , 95, 63 and 90. A one-tailed one-sample $t$ test showed that only the 'sad' response was not significantly above chance for the Down syndrome group, $t(10)=1.15, p=.3$, suggesting that children with Down syndrome were no better than chance at discriminating actions depicting 'sad' emotional states.

These scores were entered into a mixed design $2 \times 4$ repeated measures ANOVA. There was a significant main effect for Group (Down syndrome or typically developing) $F(1,2 \mathrm{l})$ 
$=6.2, p=.02$, with typically developing children outperforming Down syndrome children, and Emotion $F(3,63)$ $=50.994, p<.001$ but no significant interaction between group and emotion, suggesting that overall both groups of children showed a similar 'pattern' of performance, though at a lower level of overall accuracy.

\section{Discussion}

While children with Down syndrome are able to identify the emotional states portrayed by point-light actors at above chance levels, their performance falls short of that of typically developed children. There are two possible interpretations of this result. First, children with Down syndrome may suffer from a selective impairment in the visual perception of the emotional states of other people. Kasari et al. (2001) reported that children with Down syndrome (approx 4 years of mental age) were able to recognise emotions as well as mental aged matched children with mental retardation and typical children; however they were less accurate in labelling an emotion and in identifying emotions from a context based story. In particular, children with Down syndrome had significantly poorer performance in identifying the emotions of anger and fear in comparison with happy and sad.

Another possibility is that while children with Down syndrome are able to perform simple discriminations with point-light displays, such as the person-object discrimination task from Experiment 1, they are not able to perform more complex discriminations with point-light displays. The results of this experiment cannot differentiate between these two possible interpretations. To address this limitation, another complex perceptual discrimination task, described in Experiment 3, was developed in which children discriminated between different types of gaits rather than different types of emotions. If performance in the gait discrimination task mirrors performance in the emotion discrimination task, then we can conclude that children with Down syndrome are impaired in the performance of complex visual motion discriminations.

\section{Experiment 3}

\section{Typical vs atypical gait}

The purpose of this experiment was two fold. First, in conjunction with the results of the previous studies, the results of this experiment will indicate whether children with Down syndrome are compromised in their perception of complex point-light displays. Second, this experiment was also designed to indicate whether the motor difficulties associated with Down syndrome (see Welsh \& Elliott, 2000 for review) might alter the visual perception of action in individuals with Down syndrome.

\section{Method}

\section{Stimulus construction}

A 3-D VICON motion capture system was used to capture or measure the motions of various actors. To create displays of different gaits, eight children (four typically developing and two children with cerebral palsy and two children with Down syndrome) were asked to walk along a walkway at a comfortable speed. The children had reflective markers attached to the major joints of their bodies. These markers were then processed to create movies of 'point-light' displays against a dark background similar to that described in Experiment 1.

\section{Procedure}

A series of training displays was created to familiarise all participants with the differences between typical and atypical gait. All participants were first trained using digital videos (full colour and form) that showed walking patterns of both typically developing children and children with developmental disabilities (i.e., Cerebral Palsy, Down syndrome). Children were instructed on the differences between typical and atypical gait by using simple characteristics - i.e., the typical gait was identified as being 'regular' and the atypical gait was identified as being either 'not regular' or 'funny'. Several training videos were shown to the children until they were able to identify the differences $90 \%$ of the time. The typically developing children were able to discriminate the differences in the gaits within 4-5 trials. The children with Down syndrome required additional training and generally required 10-15 trials before they could discriminate at the level required.

In the subsequent experimental trials, all participants viewed 4 point light displays of gaits from typical children and 4 point light displays of uncoordinated gait from children with Cerebral Palsy and Down syndrome. Each stimulus was 10 seconds in duration. The stimuli were presented in a random order. The instructions asked children to verbally report whether each point-light defined person had a 'funny' walk or 'regular' walk.

\section{Results}

An individual accuracy score was calculated for each participant. A point was given for each trial in which a correct response was given, yielding scores with a range from 0 to 8 with a mean of 4 expected by chance. As depicted in Figure 6 , the average accuracy score was 4.8 for the children with Down syndrome and 6.3 for the typically developing children. An independent t-test was computed to determine if the responses of the two groups differed from one another. The responses of the two groups were significantly different, $t(19)=3.349, p=.004$. The accuracy scores for each group were then compared with chance performance with a one-tailed one-sample t test. The Down syndrome group did not perform significantly above chance: $t(9)=2.1, p$ $=.07$, whereas the typically developing group did perform significantly above chance: $t(11)=7.5, p<.0001$. 


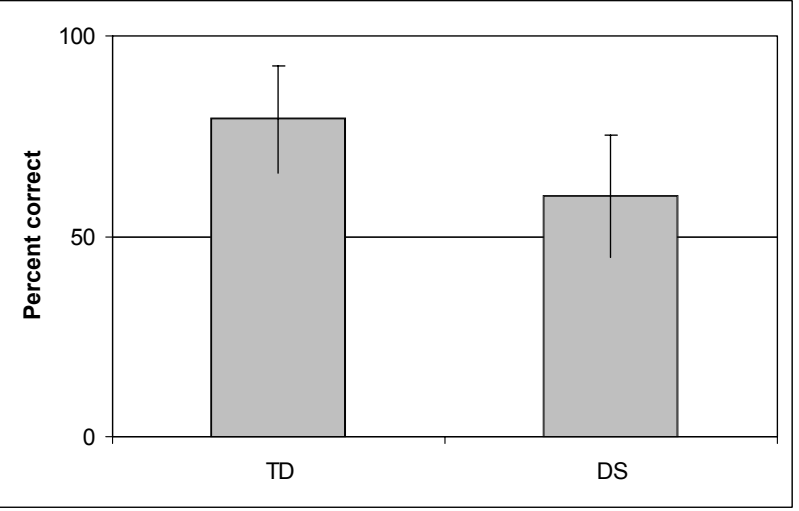

Figure 6. The results of Experiment 3. Typically developing children (TD) and children with Down syndrome (DS) discriminated normal from atypical gaits. Performance accuracy is plotted. Error bars depict standard deviation.

\section{Discussion}

The results of this study suggest that children with Down syndrome cannot reliably discriminate normal from atypical gaits when those actions are depicted as point-light displays. The fact that children with Down syndrome could learn to discriminate normal from atypical gaits during the training session (even though they required more trials than typically developing children to achieve above chance performance) is important. During training, participants viewed unaltered videotapes of children producing normal and typical gaits. These full colour videos depicted all of the form and motion cues present during real-world conditions. Since children with Down syndrome could discriminate typical from atypical gaits under these conditions, we can conclude that the use of discrimination tasks, per se, was not the cause of the performance decrement found during the experimental trials. Instead, we can conclude that children with Down syndrome are impaired in the perception of complex visual motion cues; specifically, the dynamic cues that enabled typically developing children to discriminate typical from atypical gaits.

\section{General discussion}

Relatively little research has examined the perceptual-motor abilities of children with Down syndrome. The visual perception of human action is a fundamental perceptual ability that enables individuals to have rich social interactions. Thus, the primary aim of this study was to determine whether children with Down syndrome, like typically developing children, can accurately perceive the actions of other people. Numerous studies have concluded that the visual analysis of action is an inherently perceptual-motor task (e.g., Shiffrar \& Pinto, 2002). Therefore, the study of action perception in children with Down syndrome provides an important window into the nature and extent of their perceptual-motor impairments.

In Experiment 1, typically developing children and children with Down syndrome were asked to differentiate between moving people and objects in point-light displays. Task per- formance indicates that children with Down syndrome are able to perform this basic perceptual discrimination. Thus it appears that the visual systems of children with Down syndrome have sufficiently intact visual motion processing capabilities to allow for above chance performance in simple motion discrimination tasks. Nonetheless, superior task performance by typically developing children relative to children with Down syndrome suggests that the mechanisms underlying action perception in children with Down syndrome may be compromised.

In Experiment 2, the visual perception of emotion from human action was examined in typically developing children and children with Down syndrome. Previous studies indicate that adult observers can determine an individual's emotion state from bodily motion cues alone (Dittrich et al., 1996; Heberlein et al., 2004; Kapur, Kapur, Virji-Babul et al., 2005). The current results indicate that typically developing children aged 4 to 8 are also quite capable of perceiving emotion from point-light defined actions. Performance by children with Down syndrome in our emotion perception task was above chance but significantly below that of typically developing children. In contrast to the popular view of children with Down syndrome as socially competent, there is emerging evidence that individuals with Down syndrome have difficulties in interpreting social and emotional cues and understanding mental states (see Iarocci et al., 2006). Previous studies examining emotion perception in Down syndrome have relied upon static, face or face-like stimuli (i.e., Wishart \& Pitcairn, 2000; Kasari, Freeman \& Hughes, 2001). The results of these studies suggest that individuals with Down syndrome are impaired in perception of emotion from static facial expressions. Obviously, normal social interactions also require the accurate perception of bodily action. The results of Experiment 2 suggest that children with Down syndrome have difficulty accurately perceiving the emotional signals normally generated by moving people.

Experiment 3 examined two different reasons why children with Down syndrome might exhibit performance decrements in the perception of emotion from point-light displays. One possibility is that children with Down syndrome are selectively compromised in the perception of emotion. A second possibility, which need not be contradictory, is that children with Down syndrome demonstrate general perceptual limitations in their visual analyses of complex moving stimuli. To test these two possibilities, typically developing children and children with Down syndrome were asked to discriminate normal from atypical gaits. For the first time in this series of experiments, performance levels by children with Down syndrome fell to chance levels. Specifically, these children were not able to differentiate normal from atypical gaits depicted in point-light displays. Importantly, children with Down syndrome were able to discriminate normal from atypical gaits when those gaits were depicted in regular videos. Since such videos provide bodily form cues that are unavailable in point-light displays, this combination of results suggests that children with Down syndrome have difficulties perceiving and interpreting complex 
motion stimuli. It appears that whenever the perception of emotion and gait requires complex motion analyses, performance by children with Down syndrome drops.

\section{Implications for intervention}

The results from this study add to a growing body of evidence for a need to re-conceptualise the basis of early intervention models for infants and children with Down syndrome. Traditional early intervention models that take a 'bottom-up' approach in which specialised professionals (e.g., Physiotherapists, Occupational Therapists) focus on isolating and treating specific motor based components (i.e., muscle tone, balance, etc) have not been effective in improving long term motor outcomes (e.g., Mahoney, 2004). New models of assessment (see Jobling \& VirjiBabul, 2004) and intervention are needed therefore, that focus on enhancing early perceptual-motor competencies in infants with Down syndrome.

There is increasing evidence that infants with Down syndrome show differences in attending to and processing information early in their development. For example, infants engage in significantly fewer social referencing looks (i.e., pointing, eye-gazing, and joint attending) that guide attention and action (Kasari, Freeman, Mundy \& Sigman, 1995; Landry \& Chapieski, 1990), manipulate toys less often, tend to hold toys and show more passive behaviour in response to their mothers' attention direction behaviours (Landry \& Chapieski, 1989). These early difficulties in attending, processing information and physically manipulating toys may significantly impair the child's ability to actively explore and make sense of their environment and impede the learning process of extracting salient information, in both social and physical contexts.

Iarocci, Virji-Babul and Reebye (2006) have recently proposed a transdisciplinary model that moves away from the traditional emphasis on facilitating gross and fine motor skills within a developmental framework. Instead, the goal is to nurture and shape the development of interpersonal skills and social competence. Early perceptual-motor competencies (such as eye gaze and joint attention to objects, people and the environment) are taught within a play context to maximise the quality of early dyadic interactions between infants with Down syndrome and their parents. Interventions are designed to target the specific domains (e.g., motor, language) within the broader and integrated context of social competence goals.

Further research is needed to develop and evaluate the impact of intervention programs designed to improve long term functional outcomes of engagement in meaningful physical and social activities and increasing participation in typical life experiences.

\section{Acknowledgements:}

This study was funded by the Queen Alexandra Foundation for Children's Health. We thank all the children and parents who participated in the study and the Selkirk Montessori School, Victoria, B.C. for their support. We also thank
Drs. Anne Jobling and Jennifer Wishart for their valuable feedback and comments.

\section{Correspondence}

Naznin Virji-Babul - Down Syndrome Research Foundation, 1409 Sperling Avenue, Burnaby, BC・naznin@dsrf.org.

\section{References}

Blake, R., Turner, L.M., Smoski, M.J., Pozdol, S.L. \& Stone, W.L. (2003). Visual recognition of biological motion is impaired in children with autism. Psychological Science, $14,151-157$.

Booth, A., Pinto, J. \& Bertenthal, B. (2002). Perception of the symmetrical patterning of human gait by infants. Developmental Psychology, 38, 554-563.

Bruininks, R.H., Woodcock, R.W., Weatherman R.E. \& Hill, B.K. (1996). Scales of Independent Behaviour-Revised. Riverside Publishing Company.

Carr, J. (1970). Mental and motor development in young Mongol children. Journal of Mental Deficiency Research, 14, 205-220.

Charlton, J., Ibsen, E. \& Lavelle, B.M. (2000). Control of manual skills in children with Down syndrome. In D.J. Weeks, R. Chua \& D. Elliott (Eds.), Perceptual-motor behavior in Down syndrome (pp. 25-48). Champaign, IL: Human Kinetics.

Cole, J. (1995). Pride and a daily marathon. Cambridge, MA: The MIT Press.

Dittrich, W.H., Troscianko, T., Lea, S.E.G. \& Morgan, D. (1996). Perception of emotion from dynamic point-light displays represented in dance. Perception, 25, 727-738.

Flint, J. (1999). The genetic basis of cognition. Brain, 122 (Pt 11), 2015-2032.

Fox, R. \& McDaniel, C. (1982). The perception of biological motion by human infants. Science, 218, 486-487.

Grezes, J. \& Decety, J. (2001). Functional anatomy of execution, mental simulation, observation, and verb generation of actions: a meta-analysis. Human Brain Mapping, 21, 1-19.

Grossman, E.D. \& Blake, R. (2002). Brain Areas Active during Visual Perception of Biological Motion. Neuron, $35,1167-1175$.

Haley, S.M., (1986). Postural reactions in infants with Down syndrome: Relationship to motor milestone development and age. Physical Therapy, 66, 17-22.

Harris, S.R. (1985). Neuromotor development of infants with Down syndrome. Developmental Medicine and Child Neurology, 27, 99-100.

Heberlein, A.S., Adolphs, R., Tranel, D. \& Damasio, H. (2004). Cortical regions for judgments of emotions and personality traits from point-light walkers. Journal of Cognitive Neuroscience, 16, 1143-1158.

Henderson, S.E., Morris, J. \& Frith, U. (1981). The motor deficit of Down's syndrome children: a problem of timing? Journal of Child Psychology and Psychiatry, 22, 233-245.

Iarocci, G., Virji-Babul, N. \& Reebye, P. (2006). The Learn at Play Program (LAPP): Merging family, developmental research, early intervention and policy goals for children 
with Down syndrome. Journal of Policy and Practice in Intellectual Disabilities, 3(1), 11-21.

Jobling, A. \& Virji-Babul, N. (2004). Down Syndrome: Play, Move and Grow. Vancouver: Down Syndrome Research Foundation.

Johansson, G. (1973). Visual perception of biological motion and a model for its analysis. Perception and Psychophysics, $14,201-211$

Johansson, G. (1975). Visual motion perception. Scientific American, 232(6), 76-88.

Kapur, A., Kapur A., Virji-Babul, N., Tzanetakis, G. \& Dries sen, F. (2005). Gesture-Based Affective Computing on Motion Capture Data. ACII 2005, LNCS 3784, pp. 1-7.

Kasari, C., Freeman, S.F. \& Hughes, M.A. (2001). Emotion recognition by children with Down syndrome. American Journal of Mental Retardation, 106, 59-72.

Kasari, C., Freeman, S., Mundy, P. \& Sigman, M. D. (1995). Attention Regulation by Children with Down-Syndrome - Coordinated Joint Attention and Social Referencing Looks. American Journal on Mental Retardation, 100, 128-136.

Landry, S.H. \& Chapieski, M.L. (1989). Joint attention and Infant Toy Exploration: Effects of Down syndrome and prematurity. Child Development, 60, 103-118

Landry, S.H. \& Chapieski, M. L. (1990). Joint attention of six-month-old Down syndrome and preterm infants: I. Attention to toys and mother. American Journal of Mental Retardation, 94, 488-498.

Latash, M.L. (2000). Motor coordination in Down syndrome: The role of adaptive changes. In Weeks D.J., Chua R., Elliott D. (Eds.). Perceptual-Motor Behavior in Down Syndrome, pp. 199-223, Human Kinetics: Urbana, IL.

Loula, F., Prasad, S., Harber, K. \& Shiffrar, M. (2005). Recognizing people from their movement. Journal of Experimental Psychology: Human Perception \& Performance, 31, 210-220.

Mahoney, G., Robinson, C. \& Perales, F. (2004). Infants and Young Children. In Early Motor Intervention: The Need for New Treatment Paradigms (17 ed., pp. 291-300). Lippincott Williams \& Wilkins, Inc.

Moore, D. G., Hobson, R. \& Lee. A. (1997). Components of person perception: An investigation with autistic, nonautistic retarded and typically developing children and adolescents. British Journal of Developmental Psychology, $15,401-423$.

Nadel, L (1999). Down syndrome in cognitive neuroscience perspective. In H. Tager-Flusberg (Ed.), Neurodevelopmental Disorders (pp. 197-221). Cambridge, MA: MIT Press

Nokelainen, P. \& Flint, J. (2004). Genetic effects on human cognition: lessons from the study of mental retardation syndromes. Journal of Neurology, Neurosurgery and Psychiatry, 72, 287-296.

Palisano, R.J., Walter, S.D., Russell, D.J., Rosenbaum P.L., Gemus, M., Galuppi, B.E. et al. (2001). Gross motor function of children with Down syndrome: creation of motor growth curves. Archives of Physical Medicine and Rehabilitation, 82(4), 494-500.
Pavlova, M., Krageloh-Mann, I., Sokolov, A. \& Birbaumer, N. (2001) Recognition of point-light biological motion displays by young children. Perception, 30, 925-933

Pollick, F.E., Lestou, V., Ryu, J. \& Cho, S. (2002). Estimating the efficiency of recognizing gender and affect from biological motion. Vision Research, 42, 2345-2355.

Savelsbergh, G., van der Kamp, J., Ledebt, A. \& Planinsek, T. (2000). Information-movement coupling in children with Down syndrome. In D.J. Weeks, R. Chua \& D. Elliott (Eds.), Perceptual-Motor Behavior in Down Syndrome (pp. 251-276). Champaign, IL: Human Kinetics.

Shiffrar, M. \& Pinto, J. (2002). The visual analysis of bodily motion. Common mechanisms in perception and action: Attention and Performance, Vol. XIX. (Prinz, W. \& Hommel, B., Eds.). Oxford: Oxford University Press, 381-399.

Shumway-Cook, A. \& Woollacott, M.H. (1985). Dynamics of postural control in the child with Down syndrome. Physical Therapy, 65, 1315-1322.

Spiker, D. \& Hopmann, M.R. (1997). The effectiveness of early intervention for children with Down syndrome. In M.J. Guralnick (Ed.), The Effectiveness of Early Intervention (pp. 271-305). Baltimore, MD: Brookes.

Stevens, J.A., Fonlupt, P., Shiffrar, M. \& Decety, J. (2000). New aspects of motion perception: Selective neural encoding of apparent human movements, NeuroReport, $11,109-115$.

Virji-Babul, N. \& Brown, M. (2004). Stepping over obstacles: anticipatory modifications in children with and without Down syndrome. Experimental Brain Research, 159, 487-490.

Webber, A., Virji-Babul, N., Edwards, R. \& Lesperance, M. (2004). Stiffness and postural stability in adults with Down syndrome. Experimental Brain Research, 155, 450-458.

Welsh, T.N. \& Elliott, D. (2000). Preparation and control of goal-directed limb movements in persons with Down Syndrome. In D.J. Weeks, R. Chua \& D. Elliott (Eds.), Perceptual-Motor Behavior in Down Syndrome (pp. 4970). Champaign, IL: Human Kinetics.

Wilson, M. \& Knoblich, G. (2005). The case for motor involvement in perceiving conspecifics. Psychological Bulletin, 131, 460-473.

Wishart, J.G. \& Pitcairn, T.K. (2000). Recognition of identity and expression in faces by children with Down syndrome. American Journal on Mental Retardation, $105,466-479$. 